\title{
2 p core-level binding energies of size-selected free silicon clusters: Chemical shifts and cluster structure
}

\author{
M. Vogel,${ }^{1}$ C. Kasigkeit, ${ }^{1,2}$ K. Hirsch, ${ }^{1,2}$ A. Langenberg, ${ }^{1,2}$ J. Rittmann,,${ }^{1,2}$ V. Zamudio-Bayer, ${ }^{1,2}$ A. Kulesza, ${ }^{3}$ R. Mitrić, ${ }^{3}$ \\ T. Möller, ${ }^{2}$ B. v. Issendorff, ${ }^{4}$ and J. T. Lau ${ }^{1, *}$ \\ ${ }^{1}$ Institut für Methoden und Instrumentierung der Forschung mit Synchrotronstrahlung, \\ Helmholtz-Zentrum Berlin für Materialien und Energie GmbH, Albert-Einstein-Straße 15, 12489 Berlin, Germany \\ ${ }^{2}$ Institut für Optik und Atomare Physik, Technische Universität Berlin, Hardenbergstraße 36, 10623 Berlin, Germany \\ ${ }^{3}$ Fachbereich Physik, Freie Universität Berlin, Arnimallee 14, 14195 Berlin, Germany \\ ${ }^{4}$ Fakultät für Physik, Universität Freiburg, Stefan-Meier-Straße 21, 79104 Freiburg, Germany
}

(Received 23 March 2012; published 25 May 2012)

\begin{abstract}
The $2 p$ core-level electron binding energies of size-selected silicon cluster ions have been determined from soft $x$-ray photoionization efficiency curves. Local chemical shifts and global charging energy contributions to the $2 p$ binding energy can be separated, because core-level and valence-band electron binding energies exhibit the same inverse radius dependence. The experimental $2 p$ binding energy distributions show characteristic size-specific patterns that are well reproduced by the corresponding electronic density of states obtained from density functional theory modeling. These results demonstrate that $2 p$ binding energies in silicon clusters are dominated by initial state effects, i.e., by the interaction with the local valence electron density, and can thus be used to corroborate structural assignments.
\end{abstract}

DOI: 10.1103/PhysRevB.85.195454

PACS number(s): $61.46 . \mathrm{Bc}, 36.40 . \mathrm{Mr}, 33.15 . \mathrm{Ry}, 73.22 .-\mathrm{f}$

\section{INTRODUCTION}

$\mathrm{X}$-ray photoelectron spectroscopy is a standard technique in the investigation of the electronic structure of surfaces, adsorbates, thin films, deposited clusters, and nanocrystals. ${ }^{1-4}$ Its application to free particles like size-selected clusters, nanoparticles, or complexes should allow to study their unperturbed properties, but this approach is still hindered by considerable technical challenges. While photoelectron spectroscopy of size-selected cluster anions in the visible and ultraviolet spectral range can be performed with laboratory lasers, the access to deeper valence bands or core levels requires photon energies in the extreme ultraviolet to soft x-ray range, and synchrotron radiation facilities or free-electron lasers as photon sources. Since the photon flux of conventional undulator radiation is typically 3-5 orders of magnitude lower than that of lasers, core-level photoelectron spectroscopy on gas phase clusters has so far only been performed on neutral cluster beams with a broad size distribution, ${ }^{5}$ or on very large nanoparticles. ${ }^{6}$ However, these approaches do not allow to observe detailed finite size effects. Recently, first free-electron laser vacuum ultraviolet and soft $\mathrm{x}$-ray photoelectron spectra of size-selected clusters have been reported. ${ }^{7}$ Still, photoelectron spectroscopy on a beam of size-selected clusters remains challenging even with the increased photon flux of a free-electron laser because of the inherently low target density. In principle, the same also applies to x-ray absorption spectroscopy. In this case, however, ion traps can be used to increase the target density, which permits to perform x-ray absorption ${ }^{8-14}$ and $\mathrm{X}$-ray magnetic circular dichroism ${ }^{15,16}$ spectroscopy of sizeselected atomic, molecular, and cluster ions with synchrotron radiation. Such experiments allow to study the electronic and magnetic properties of free particles, and give access to core-level and valence electron binding energies as well as highest occupied-lowest unoccupied molecular orbital gaps. ${ }^{17}$

Of particular interest in this respect are silicon clusters, which exhibit strongly size dependent electronic and geometric properties. ${ }^{18-21}$ Here, we demonstrate that $2 p$ binding energy spectra can be extracted from core-level photoionization efficiency curves of size-selected free silicon clusters. In combination with density functional theory calculations, these binding energy spectra yield information about the intracluster electron density distribution and its relation to geometric structure.

\section{EXPERIMENTAL METHOD}

Details of the experimental setup are described elsewhere. ${ }^{11}$ In brief, cationic silicon clusters are produced in a magnetron gas aggregation source by argon sputtering of a high-purity silicon target. The silicon cluster cations are guided into high vacuum by a radio-frequency hexapole ion guide, mass selected by a quadrupole mass filter, and accumulated in a linear quadrupole ion trap. The confined cluster ions are irradiated by a monochromatic soft x-ray beam at BESSY II undulator beamline U125/2-SGM. Following x-ray absorption at the silicon $\mathrm{L}_{2,3}$ edges, the $2 p$ core-excited state decays in a nonradiative process with the emission of Auger electrons, ${ }^{22,23}$ which results in multiply charged cluster ions that may further undergo fragmentation or evaporation. For ion yield spectroscopy, these product ions are detected by a reflectron time-of-flight mass spectrometer as the photon energy is scanned across the silicon $2 p$ core level binding energy with a step size and resolution of 50-100 meV, as shown in Fig. 1 for $\mathrm{Si}_{11}^{+}$.

\section{EXPERIMENTAL CORE-LEVEL ELECTRON BINDING ENERGIES FROM PHOTOION YIELD SPECTRA}

In contrast to transition metal clusters, ${ }^{10-15}$ silicon clusters show complex photofragmentation patterns ${ }^{24-26}$ not only in valence band, but also in $2 p$ core-level photoexcitation. For silicon $2 p$ excitation, the resulting partial ion yield curves allow to distinguish between direct and resonant 


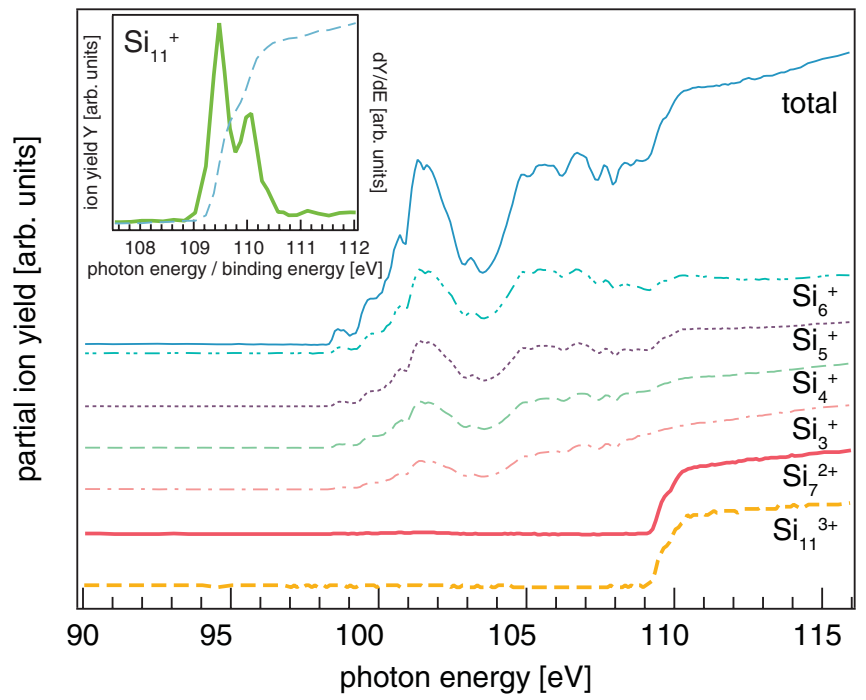

FIG. 1. (Color online) Normalized partial ion yield spectra of $2 p$ excited $\mathrm{Si}_{11}^{+} \cdot \mathrm{Si}_{11}^{3+}$ (thick dashed line) and $\mathrm{Si}_{7}^{2+}$ (thick solid line) originate from direct $2 p$ photoionization and give access to the $2 p$ core level binding energies, while $\mathrm{Si}_{n}^{+}, n=3-6$ product ions originate from resonant excitation. Inset: $2 p$ photoionization efficiency curve (dashed line) and its derivative (solid line) for $\mathrm{Si}_{11}^{+}$.

photoionization processes. These curves are shown in Fig. 1 for $\mathrm{Si}_{11}^{+}$parent clusters. Here, the $\mathrm{Si}_{11}^{3+}$ and $\mathrm{Si}_{7}^{2+}$ photoion yield curves exhibit a sharp threshold at $\approx 109.5 \mathrm{eV}$, indicating that these product ions originate from direct $2 p$ core level photoionization, while $\mathrm{Si}_{n}^{+}, n=3-6$ are produced already at $\approx 10 \mathrm{eV}$ lower photon energy, therefore resulting from resonant $2 p$ excitation. This clear separation of direct and resonant photoionization processes in the ion yield curves is surprising, but follows from the possible excitation and relaxation channels. The silicon $2 p$ level is a shallow core-level situated below the $3 s$ and $3 p$ valence levels. Following direct $2 p$ photoionization of $\mathrm{Si}_{n}^{+}, \mathrm{Si}_{n}^{2+}$ can therefore only relax via a single $L V V$ Auger decay, which limits the final charge state to $\mathrm{Si}_{n}^{3+}$. The maximum internal energy of this electronically excited $\mathrm{Si}_{n}^{3+}$ product ion is obtained when removing two electrons from the bottom of the valence band in the Auger decay, and equals twice the valence band width. Although this internal energy of $\approx 24 \mathrm{eV}$ (see Ref. 27) would be sufficient to overcome the fourth ionization potential, the corresponding double excited state does not couple efficiently to further ionization, but leads to fragmentation. For small silicon clusters $(n \leqslant 10)$, asymmetric fission with the emission of $\mathrm{Si}^{+}$is the preferred channel for both doubly and triply charged clusters. Larger clusters exhibit more complex fragmentation patterns with a preference for the emission of $\mathrm{Si}_{6}^{+}, \mathrm{Si}_{7}^{+}, \mathrm{Si}_{10}^{+}$, and $\mathrm{Si}_{11}^{+} \cdot{ }^{21,24-26}$ Therefore direct $2 p$ photoionization of $\mathrm{Si}_{n}^{+}$and subsequent Auger decay produces triply charged $\mathrm{Si}_{n}^{3+}$ ions, which can decay to dicationic $\mathrm{Si}_{m}^{2+}(m<n)$ via the loss of singly charged fragments.

In resonant excitation of $\mathrm{Si}_{n}^{+}$, on the contrary, a $2 p$ core electron is promoted into unoccupied valence states with $s$ or $d$ character, again followed by a single $L V V$ Auger decay, limiting the charge state to $\mathrm{Si}_{n}^{2+}$. Again, the remaining internal energy leads to (asymmetric) fission that yields singly charged
TABLE I. List of doubly and triply charged product ions used to monitor direct $2 p$ photoionization efficiency curves for a given $\mathrm{Si}_{n}^{+}$ parent ion.

\begin{tabular}{llll}
\hline & \multicolumn{1}{c}{ Product ions } & & \multicolumn{1}{c}{ Product ions } \\
\hline $\mathrm{Si}_{9}^{+}$ & $\mathrm{Si}_{7}^{2+}, \mathrm{Si}_{6}^{2+}, \mathrm{Si}_{5}^{2+}$ & $\mathrm{Si}_{18}^{+}$ & $\mathrm{Si}_{11}^{2+}, \mathrm{Si}_{9}^{2+}$ \\
$\mathrm{Si}_{10}^{+}$ & $\mathrm{Si}_{9}^{2+}, \mathrm{Si}_{7}^{2+}, \mathrm{Si}_{6}^{2+}$ & $\mathrm{Si}_{19}^{+}$ & $\mathrm{Si}_{11}^{2+}, \mathrm{Si}_{9}^{2+}$ \\
$\mathrm{Si}_{11}^{+}$ & $\mathrm{Si}_{11}^{3+}, \mathrm{Si}_{7}^{2+}$ & & \\
$\mathrm{Si}_{12}^{+}$ & $\mathrm{Si}_{11}^{2+}$ & $\mathrm{Si}_{22}^{+}$ & $\mathrm{Si}_{22}^{3+}, \mathrm{Si}_{11}^{2+}$ \\
$\mathrm{Si}_{13}^{+}$ & $\mathrm{Si}_{13}^{3+}, \mathrm{Si}_{7}^{2+}$ & $\mathrm{Si}_{23}^{+}$ & $\mathrm{Si}_{23}^{3+}, \mathrm{Si}_{17}^{2+}, \mathrm{Si}_{13}^{2+}, \mathrm{Si}_{11}^{2+}$ \\
$\mathrm{Si}_{14}^{+}$ & $\mathrm{Si}_{14}^{3+}, \mathrm{Si}_{8}^{2+}, \mathrm{Si}_{7}^{2+}$ & $\mathrm{Si}_{24}^{+}$ & $\mathrm{Si}_{17}^{2+}, \mathrm{Si}_{13}^{2+}, \mathrm{Si}_{11}^{++}$ \\
$\mathrm{Si}_{15}^{+}$ & $\mathrm{Si}_{11}^{2+}, \mathrm{Si}_{9}^{2+}, \mathrm{Si}_{8}^{2+}, \mathrm{Si}_{7}^{2+}$ & $\mathrm{Si}_{25}^{+}$ & $\mathrm{Si}_{25}^{3+}, \mathrm{Si}_{19}^{2+}, \mathrm{Si}_{15}^{2+}, \mathrm{Si}_{11}^{2+}$ \\
$\mathrm{Si}_{16}^{+}$ & $\mathrm{Si}_{16}^{3+}, \mathrm{Si}_{11}^{2+}, \mathrm{Si}_{10}^{2+}, \mathrm{Si}_{9}^{2+}$ & $\mathrm{Si}_{26}^{+}$ & $\mathrm{Si}_{26}^{3+}, \mathrm{Si}_{19}^{++}, \mathrm{Si}_{15}^{++}, \mathrm{Si}_{11}^{++}$ \\
$\mathrm{Si}_{17}^{+}$ & $\mathrm{Si}_{11}^{2+}, \mathrm{Si}_{10}^{2+}, \mathrm{Si}_{9}^{2+}$ & $\mathrm{Si}_{27}^{+}$ & $\mathrm{Si}_{21}^{2+}, \mathrm{Si}_{19}^{2+}, \mathrm{Si}_{17}^{2+}, \mathrm{Si}_{11}^{2+}$ \\
\hline \hline
\end{tabular}

$\mathrm{Si}_{k}^{+}$ions with $k<n$. Therefore the size and charge of a photoion allows to trace back its origin to either resonant or direct core-level photoionization: In the present study, $\mathrm{Si}_{n}^{3+}$ and $\mathrm{Si}_{m}^{2+}$ ion yields for $m<n$ almost exclusively represent the $2 p$ photoionization efficiency curves of $\mathrm{Si}_{n}^{+}$. The dominant ion yield channels which have been used to obtain the direct photoionization efficiency curves are summarized in Table I. From these curves, information about binding energies and chemical shifts of the $2 p$ core level for $\mathrm{Si}_{n}^{+}$parent clusters can be obtained. This is illustrated in the inset of Fig. 1, where the $2 p$ photoionization efficiency curve and its derivative is shown for $\mathrm{Si}_{11}^{+}$. The two lines in the derivative are separated by the $2 p$ spin-orbit splitting of $0.6 \mathrm{eV}$ (see Ref. 28) and exhibit an intensity ratio of $2: 1$. These lines represent the $2 p$ electron binding energy spectrum with respect to the vacuum level and can be attributed to $2 p_{3 / 2}$ and $2 p_{1 / 2}$ core-hole states.

\section{GLOBAL AND LOCAL CONTRIBUTIONS TO CORE-LEVEL BINDING ENERGIES OF SIZE-SELECTED SILICON CLUSTERS}

An overview of the evolution of silicon $2 p$ binding energies with cluster size is shown in Fig. 2 for $\mathrm{Si}_{n}^{+}$with $9 \leqslant n \leqslant 27$. Two effects can be seen in these spectra: an overall shift to lower $2 p$ binding energy with increasing cluster size, and a size specific variation in the silicon $2 p$ binding energy spectrum. The overall shift can be attributed to the global charging energy of $\mathrm{Si}_{n}^{+}$clusters while the detailed structure of the binding energy distribution reflects local chemical shifts in the $2 p$ binding energy of different atoms in the cluster.

Within the well-known macroscopic spherical droplet model, ${ }^{29,30}$ which is successfully applied to describe valence band photoionization of size selected dielectric clusters, ${ }^{31}$ the ionization potential IP changes with the approximated cluster radius $\mathrm{R}$ as

$$
\mathrm{IP}(R)=\mathrm{IP}_{\infty}+(Z+\alpha)\left(1-\frac{1}{\epsilon_{r}}\right) \frac{e^{2}}{(R+\eta)},
$$

where $\mathrm{IP}_{\infty}$ is the bulk work function or the $2 p$ electron binding energy, $Z$ is the initial charge of the cluster, $\alpha$ is a material 


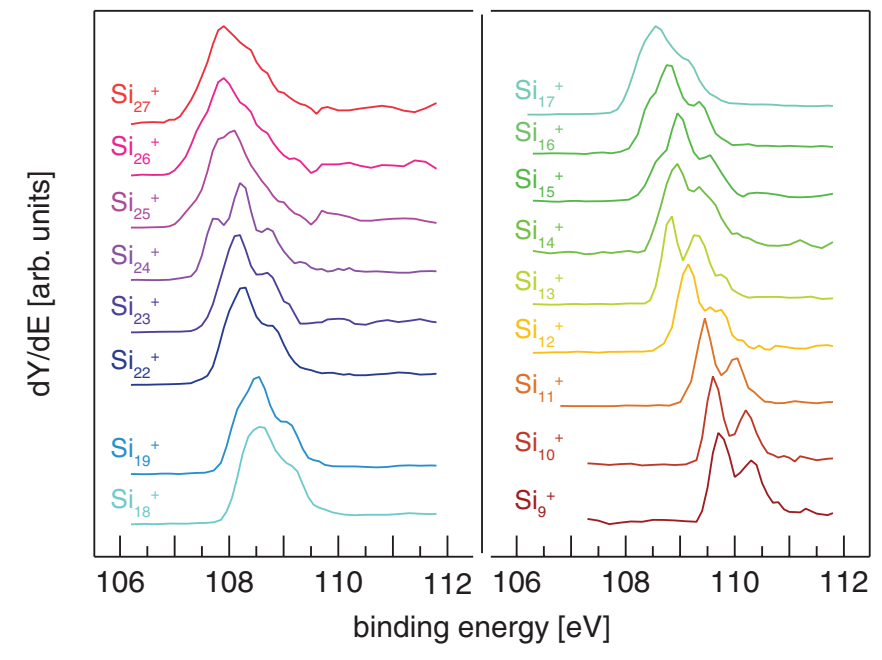

FIG. 2. (Color online) $2 p$ core level binding energy spectra of $\mathrm{Si}_{n}^{+}$clusters, reflecting global charging energy and local core-level shift contributions.

specific parameter, $e$ is the unit charge, $\epsilon_{\mathrm{r}}$ is the permittivity of the cluster, and $\eta$ is commonly interpreted as the electron spill-out. This spill-out should be smaller for cations than for anions and, for larger clusters, is small compared to the cluster radius $R$. Thus a linear change of the ionization potential with inverse radius $1 / R$ is expected within the spherical droplet model, with the same slope for both, valence and $2 p$ core level photoionization.

To show the validity of this model for core-level photoionization, $2 p$ photoionization thresholds, obtained from the average core-level binding energies of $\mathrm{Si}_{n}^{+}, n=5-92$, are plotted versus $n^{-1 / 3} \propto 1 / R$ in Fig. 3. As expected, the direct $2 p$ photoionization threshold of $106-111 \mathrm{eV}$ increases linearly with $n^{-1 / 3}$, as does the valence level photoionization threshold in the range of $8-13 \mathrm{eV}$, also included in Fig. 3. This valence level photoionization threshold was obtained with the same

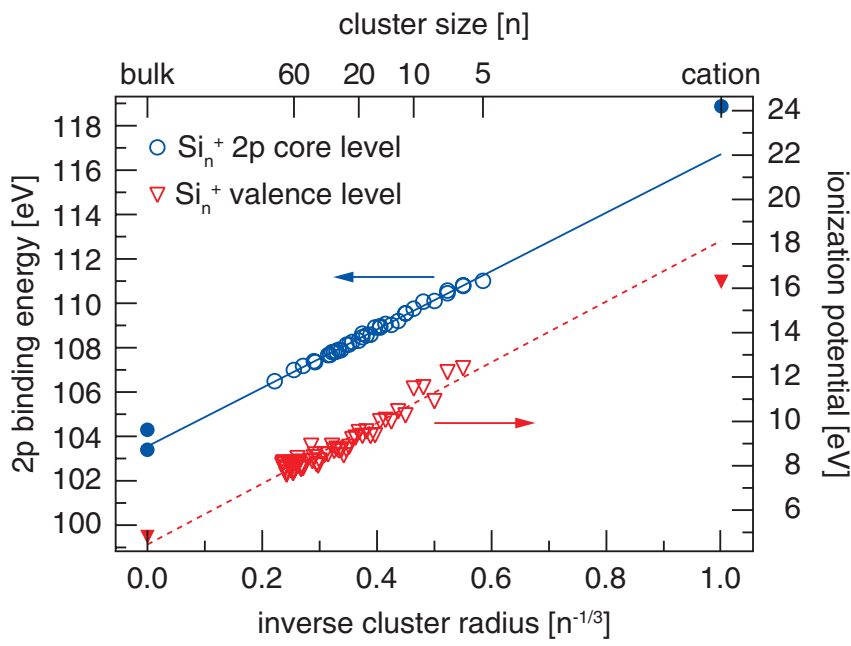

FIG. 3. (Color online) $2 p$ photoionization threshold (open circles) and ionization potential (open triangles) for $\mathrm{Si}_{n}^{+}$clusters. The macroscopic charging energy, determined from the slopes of both curves, is identical within the error bars and interpolates between atomic and bulk silicon (filled symbols), see Refs. 28,32-34. setup from photoionization efficiency curves of $\mathrm{Si}_{n}^{+}$in the vacuum ultraviolet spectral range, where the photon energy at the onset of the $\mathrm{Si}_{n}^{2+}$ signal in the photoion yield spectrum was taken as the ionization threshold of $\mathrm{Si}_{n}^{+}$. As expected, the slopes of both curves, and thus the charging energies, are identical within the error bars. This allows for the separation of global and local contributions to the size dependence of the $2 p$ core level binding energy spectra by subtracting the charging energy contribution. The remaining core-level shifts contain information on the local charge density and can be linked to the cluster geometry.

\section{DENSITY FUNCTIONAL THEORY MODELING OF CORE-LEVEL BINDING ENERGY SPECTRA}

To study the effect of cluster structure, $2 p$ binding energy spectra were modeled for $\mathrm{Si}_{n}^{+}, n=9-13$. This size range was chosen because of the absence of low-lying structural isomers ${ }^{18,19}$ that allows for the assignment of individual lines to specific sites, and thus, for geometric structure determination. Reported geometries ${ }^{20}$ of silicon clusters were reoptimized ${ }^{35}$ with density functional theory (DFT) as implemented in TURBOMOLE, ${ }^{36,37}$ using the Becke three parameter exchange and Lee-Yang-Parr correlation (B3LYP) hybrid functional ${ }^{38,39}$ with the triple- $\zeta$ valence plus polarization (def2-TZVP) atomic basis set. ${ }^{40}$ In addition, global optimization using simulated annealing coupled to molecular dynamics (MD) simulations was performed for $\mathrm{Si}_{12}^{+},{ }^{35}$ followed by optimization with gradient based techniques and characterization of the stationary points by vibrational frequency calculation. Gradients for MD simulations have been obtained using the Becke exchange and Perdew or Lee-Yang-Parr correlation (BP86, BLYP) functionals $\mathrm{s}^{39,41,42}$ with the split valence plus polarization (def2-SVP) basis set ${ }^{40}$ and employing the resolution of identity approximation.

Ground state $2 p$ core-level electron binding energies were obtained from Kohn-Sham eigenvalues of the corresponding orbitals. ${ }^{43}$ Final state spin-orbit splitting was modeled in the calculated spectra by a duplicated ground state density of states, separated by the silicon $2 p$ spin-orbit splitting of $0.6 \mathrm{eV},{ }^{28}$ and weighted with the $j$-degeneracy of $2 p_{3 / 2}$ and $2 p_{1 / 2}$ core hole states. The resulting individual lines were broadened by $290 \mathrm{meV}$ full width half maximum Gaussian profiles and calculated spectra were shifted by $<500 \mathrm{meV}$ to match the experimental data.

\section{CORE-LEVEL SHIFTS, LOCAL VALENCE ELECTRON DENSITY, AND CLUSTER GEOMETRY}

In Fig. 4, the experimental and theoretical results are compared for $\mathrm{Si}_{n}^{+}, n=9-13$. The calculated $2 p$ core-level electron binding energy spectra are in very good agreement with the experimental data, underlining the close relationship of electronic and geometric structure. Since final state effects beyond the simulated $2 p$ spin-orbit splitting in the presence of the core hole were not accounted for in the calculations, this good agreement implies that the experimental spectra are dominated by initial state effects.

For $\mathrm{Si}_{13}^{+}$, three lines are resolved in the experimental $2 p$ binding energy spectrum. In its lowest energy structure, ${ }^{20}$ the 


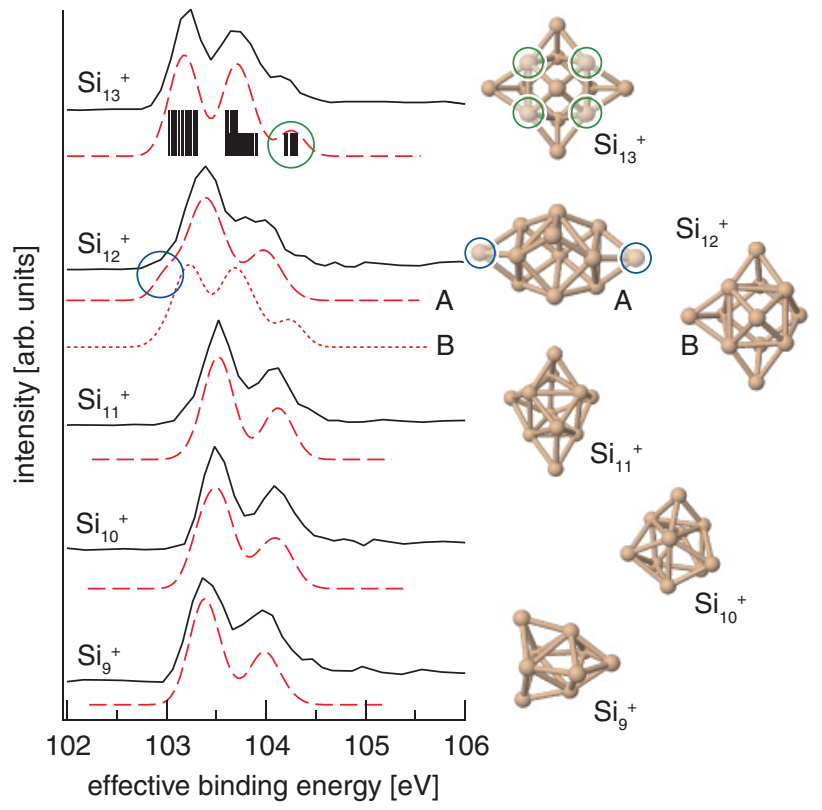

FIG. 4. (Color online) Experimental (solid lines) and modeled (dashed lines, sticks) $2 p$ electron binding spectra for $\mathrm{Si}_{n}^{+}, n=9-13$, plotted versus effective binding energy without charging energy contribution. For $n=12$, different spectra are shown for isomers A (dashed line) and B (dotted line), see Ref. 20.

ground-state $2 p$ electron binding energies of $\mathrm{Si}_{13}^{+}$appear in two groups which are separated by a gap of $0.3 \mathrm{eV}$ in each set of $2 p_{3 / 2}$ and $2 p_{1 / 2}$ lines, represented by sticks in Fig. 4. These two different groups of binding energies can be assigned to different sites within the cluster: the $2 p$ levels with lower binding energies belong to the nine outermost silicon atoms with lower coordination number, while the stronger bound $2 p$ levels are localized on the four silicon atoms with higher coordination number. These chemical shifts can be correlated with calculated local differences in the electron density: consistent with $2 p$ core-level shifts of surface components observed for single-crystalline silicon, ${ }^{28,44,45}$ a decreased local electron density causes an increased $2 p$ binding energy in $\mathrm{Si}_{13}^{+}$.

For $\mathrm{Si}_{12}^{+}$, a global optimization procedure was used to obtain the ground state structure labeled isomer A in Fig. 4. Its calculated $2 p$ binding energies agree well with the experimental spectrum. Both, experimental and theoretical $2 p$ binding energy spectra of $\mathrm{Si}_{12}^{+}$exhibit a weak shoulder at low electron binding energy, which can be assigned to electrons located at the two outermost atoms in this cluster. $\mathrm{As}$ in $\mathrm{Si}_{13}^{+}$, low coordination numbers correspond to low $2 p$ electron binding energy in $\mathrm{Si}_{12}^{+}$. At the B3LYP and def2-TZVP level, ${ }^{38-41}$ isomer $\mathrm{A},{ }^{35}$ whose structure is similar to the one obtained by De et al., ${ }^{46}$ is $190 \mathrm{meV}$ more stable than the lowest energy isomer of $\mathrm{Si}_{12}^{+}$reported previously ${ }^{20}$ and labeled isomer $\mathrm{B}$ in Fig. 4. In contrast to isomer $\mathrm{A}$, the modeled spectrum for isomer $\mathrm{B}^{20}$ does not fit the experimental $2 p$ binding energy spectrum, demonstrating the sensitivity of core level binding energies to cluster geometry in this case. The structural assignment performed here for $\mathrm{Si}_{12}^{+}$is supported by the calculated infrared spectrum of isomer $\mathrm{A},{ }^{35}$ which agrees with the experimental spectrum reported by Lyon et al. ${ }^{20,35}$

For $\mathrm{Si}_{11}^{+}, \mathrm{Si}_{10}^{+}$and, to a lesser degree, for $\mathrm{Si}_{9}^{+}$, the spectra indicate a less heterogeneous electron distribution in each cluster, since no strong core level shifts are visible. In particular, the spectrum of $\mathrm{Si}_{10}^{+}$, which is predicted to form a compact tetracapped trigonal prism, ${ }^{20}$ shows two well separated $2 p_{3 / 2}$ and $2 p_{1 / 2}$ lines. This hints at a rather homogeneous electron density distribution within this cluster, in agreement with its predicted compact tetracapped trigonal prism structure. ${ }^{20}$ In contrast, prolate structures ${ }^{18,19}$ in the size range of $n=14-27$ contain an increasing number of inequivalent sites, leading to increasingly broader lines in Fig. 2 where the $2 p$ spin-orbit splitting can no longer be resolved.

\section{CONCLUSION}

In conclusion, photoionization efficiency spectroscopy at shallow core levels of size-selected free clusters can give access to inner-shell electron binding energy spectra. The observed core-level shifts are sensitive to variations in the local electron density distribution and can be used to corroborate structural assignments in special cases. The results show that high-resolution $\mathrm{x}$-ray photoelectron spectroscopy is a very interesting tool for the study of size-selected free clusters, complexes, or nanoparticles, and can serve as a motivation to overcome the technical challenges that still prevent routine application of x-ray photoelectron spectroscopy to these systems.

\section{ACKNOWLEDGMENTS}

This work was supported by DFG Grant LA 2398/5-1 within FOR 1282. Beam time for this project was granted by BESSY II at beamlines U125/2-SGM and U125/2-NIM. BESSY II is operated by Helmholtz-Zentrum Berlin. Technical assistance and user support by R. Follath, G. Reichardt, H. Pfau, and P. Baumgärtel is gratefully acknowledged. Access to the computer cluster was provided by FOR 1282. The authors would like to thank A. Fielicke and J. T. Lyon for kindly sharing their cluster geometry data. M.V. acknowledges support by DFG SFB 508 in the early stages of this work. *tobias.lau@ helmholtz-berlin.de

${ }^{1}$ S. Svensson, N. Mårtensson, E. Basilier, P. Å. Malmqvist, U. Gelius, and K. Siegbahn, J. Electron Spectrosc. Relat. Phenom. 9, 51 (1976).

${ }^{2}$ G. K. Wertheim, S. B. DiCenzo, and D. N. E. Buchanan, Phys. Rev. B 33, 5384 (1986).
${ }^{3}$ C. McGinley, H. Borchert, M. Pflughoefft, S. Al Moussalami, A. R. B. de Castro, M. Haase, H. Weller, and T. Möller, Phys. Rev. B 64, 245312 (2001).

${ }^{4}$ H. Ogasawara, B. Brena, D. Nordlund, M. Nyberg, A. Pelmenschikov, L. G. M. Pettersson, and A. Nilsson, Phys. Rev. Lett. 89, 276102 (2002). 
${ }^{5}$ O. Björneholm, F. Federmann, F. Fössing, and T. Möller, Phys. Rev. Lett. 74, 3017 (1995).

${ }^{6}$ J. Meinen, S. Khasminskaya, M. Eritt, T. Leisner, E. Antonsson, B. Langer, and E. Rühl, Rev. Sci. Instrum. 81, 085107 (2010).

${ }^{7}$ V. Senz, T. Fischer, P. Oelssner, J. Tiggesbäumker, J. Stanzel,

C. Bostedt, H. Thomas, M. Schöffler, L. Foucar, M. Martins, J. Neville, M. Neeb, T. Möller, W. Wurth, E. Rühl, R. Dörner, H. Schmidt-Böcking, W. Eberhardt, G. Ganteför, R. Treusch, P. Radcliffe, and K.-H. Meiwes-Broer, Phys. Rev. Lett. 102, 138303 (2009).

${ }^{8}$ R. Thissen, J. M. Bizau, C. Blancard, M. Coreno, C. Dehon, P. Franceschi, A. Giuliani, J. Lemaire, and C. Nicolas, Phys. Rev. Lett. 100, 223001 (2008).

${ }^{9}$ M. C. Simon, J. R. Crespo López-Urrutia, C. Beilmann, M. Schwarz, Z. Harman, S. W. Epp, B. L. Schmitt, T. M. Baumann, E. Behar, S. Bernitt, R. Follath, R. Ginzel, C. H. Keitel, R. Klawitter, K. Kubiček, V. Mäckel, P. H. Mokler, G. Reichardt, O. Schwarzkopf, and J. Ullrich, Phys. Rev. Lett. 105, 183001 (2010).

${ }^{10}$ J. T. Lau, J. Rittmann, V. Zamudio-Bayer, M. Vogel, K. Hirsch, P. Klar, F. Lofink, T. Möller, and B. v. Issendorff, Phys. Rev. Lett. 101, 153401 (2008).

${ }^{11}$ K. Hirsch, J. T. Lau, P. Klar, A. Langenberg, J. Probst, J. Rittmann, M. Vogel, V. Zamudio-Bayer, T. Möller, and B. von Issendorff, J. Phys. B 42, 154029 (2009).

${ }^{12}$ J. T. Lau, K. Hirsch, P. Klar, A. Langenberg, F. Lofink, R. Richter, J. Rittmann, M. Vogel, V. Zamudio-Bayer, T. Möller, and B. v. Issendorff, Phys. Rev. A 79, 053201 (2009).

${ }^{13}$ J. T. Lau, K. Hirsch, A. Langenberg, J. Probst, R. Richter, J. Rittmann, M. Vogel, V. Zamudio-Bayer, T. Möller, and B. von Issendorff, Phys. Rev. B 79, 241102 (2009).

${ }^{14}$ K. Hirsch, V. Zamudio-Bayer, F. Ameseder, A. Langenberg, J. Rittmann, M. Vogel, T. Möller, B. v. Issendorff, and J. T. Lau, Phys. Rev. A (unpublished).

${ }^{15}$ M. Niemeyer, K. Hirsch, V. Zamudio-Bayer, A. Langenberg, M. Vogel, M. Kossick, C. Ebrecht, K. Egashira, A. Terasaki, T. Möller, B. v. Issendorff, and J. T. Lau, Phys. Rev. Lett. 108, 057201 (2012).

${ }^{16}$ S. Peredkov, M. Neeb, W. Eberhardt, J. Meyer, M. Tombers, H. Kampschulte, and G. Niedner-Schatteburg, Phys. Rev. Lett. 107, 233401 (2011).

${ }^{17}$ J. T. Lau, M. Vogel, A. Langenberg, K. Hirsch, J. Rittmann, V. Zamudio-Bayer, T. Möller, and B. von Issendorff, J. Chem. Phys. 134, 041102 (2011).

${ }^{18}$ M. F. Jarrold and J. E. Bower, J. Chem. Phys. 96, 9180 (1992).

${ }^{19}$ R. R. Hudgins, M. Imai, M. F. Jarrold, and P. Dugourd, J. Chem. Phys. 111, 7865 (1999).
${ }^{20}$ J. T. Lyon, P. Gruene, A. Fielicke, G. Meijer, E. Janssens, P. Claes, and P. Lievens, J. Am. Chem. Soc. 131, 1115 (2009).

${ }^{21}$ W. Qin, W.-C. Lu, L.-Z. Zhao, Q.-J. Zang, C. Z. Wang, and K. M. Ho, J. Phys.: Condens. Matter 21, 455501 (2009).

${ }^{22}$ M. H. Chen, B. Crasemann, and H. Mark, Phys. Rev. A 24, 177 (1981).

${ }^{23}$ M. O. Krause, J. Phys. Chem. Ref. Data 8, 307 (1979).

${ }^{24}$ L. A. Bloomfield, R. R. Freeman, and W. L. Brown, Phys. Rev. Lett. 54, 2246 (1985).

${ }^{25}$ Q.-L. Zhang, Y. Liu, R. F. Curl, F. K. Tittel, and R. E. Smalley, J. Chem. Phys. 88, 1670 (1988).

${ }^{26}$ A. A. Shvartsburg, M. F. Jarrold, B. Liu, Z.-Y. Lu, C.-Z. Wang, and K.-M. Ho, Phys. Rev. Lett. 81, 4616 (1998).

${ }^{27}$ J. Muscat, A. Wander, and N. Harrison, Chem. Phys. Lett. 342, 397 (2001).

${ }^{28}$ E. Landemark, C. J. Karlsson, Y.-C. Chao, and R. I. G. Uhrberg, Phys. Rev. Lett. 69, 1588 (1992).

${ }^{29}$ J. P. Perdew, Phys. Rev. B 37, 6175 (1988).

${ }^{30}$ J. Jortner, Z. Phys. D 24, 247 (1992).

${ }^{31}$ O. Björneholm, F. Federmann, F. Fössing, T. Möller, and P. Stampfli, J. Chem. Phys. 104, 1846 (1996).

${ }^{32}$ C. Sebenne, D. Bolmont, G. Guichar, and M. Balkanski, Phys. Rev. B 12, 3280 (1975).

${ }^{33}$ W. Martin and R. Zalubas, J. Phys. Chem. Ref. Data 12, 323 (1983).

${ }^{34}$ J. T. Costello, E. T. Kennedy, J.-P. Mosnier, and M. H. Sayyad, J. Phys. B 31, 677 (1998).

${ }^{35}$ See Supplemental Material at http://link.aps.org/supplemental/ 10.1103/PhysRevB.85.195454 for cluster geometry files and the calculated infrared spectrum of isomer A.

${ }^{36}$ R. Ahlrichs, M. Bär, M. Häser, H. Horn, and C. Kölmel, Chem. Phys. Lett. 162, 165 (1989).

${ }^{37}$ P. Deglmann, K. May, F. Furche, and R. Ahlrichs, Chem. Phys. Lett. 384, 103 (2004).

${ }^{38}$ A. D. Becke, J. Chem. Phys. 98, 5648 (1993).

${ }^{39}$ C. Lee, W. Yang, and R. G. Parr, Phys. Rev. B 37, 785 (1988).

${ }^{40} \mathrm{~F}$. Weigend and R. Ahlrichs, Phys. Chem. Chem. Phys. 7, 3297 (2005).

${ }^{41}$ A. D. Becke, Phys. Rev. A 38, 3098 (1988).

${ }^{42}$ J. P. Perdew, Phys. Rev. B 33, 8822 (1986).

${ }^{43}$ S. Hamel, P. Duffy, M. E. Casida, and D. R. Salahub, J. Electron Spectrosc. Relat. Phenom. 123, 345 (2002).

${ }^{44}$ E. Pehlke and M. Scheffler, Phys. Rev. Lett. 71, 2338 (1993).

${ }^{45}$ C. J. Karlsson, E. Landemark, Y.-C. Chao, and R. I. G. Uhrberg, Phys. Rev. B 50, 5767 (1994).

${ }^{46}$ S. De, S. A. Ghasemi, A. Willand, L. Genovese, D. Kanhere, and S. Goedecker, J. Chem. Phys. 134, 124302 (2011). 